\title{
ОЖИДАЕМАЯ ПРОДОЛЖИТЕЛЬНОСТЬ ЖИЗНИ ПОЖИЛЫХ В РОССИИ В ЗАВИСИМОСТИ ОТ ОБРАЗОВАТЕЛЬНОГО СТАТУСА
}

\author{
СЕРГЕЙ ШУЛЬГИН, ЮЛИЯ ЗИНЬКИНА, СЕРГЕЙ ЩЕРБОВ
}

\begin{abstract}
В России в настоящее время увеличивается доля населения в старших возрастных когортах, при этом в старшие возраста входят все более образованные слои населения. В данной работе мы анализируем различия смертности в зависимости от уровня образования в России у лии в возрастах от 50 лет. В работе оцениваются российские таблииь смертности для возрастов от 50 лет. Оиенки строятся на основе лонгитюдного обследования РМЭЗ НИУ ВШЭ для периода с 1994 по 2015 г2. $и$ учитывают волны обследования с пятой по 24-ю. Оиенки таблиц смертности делаются отдельно для мужчин и женщин. Для каждого пола помимо оценок общих таблиц смертности делаются оценки таблии смертности для групп населения с высоким и низким образовательным статусом. В группу с низким образовательным статусом отнесены индивиды, у которых продолжительность обучения составляет 10 лет и менее; в группе с высоким образовательным статусом находятся индивиды с продолжительностью обучения более 10 лет. Расчеты показывают, что ожидаемая продолжительность жизни в возрасте 50 лет для мужчин с высоким образовательным статусом на три года больше, чем для мужчин с низким образовательным статусом. Для женщин с высоким образовательным статусом ожидаемая продолжительность жизни в возрасте 50 лет на 4,4 года превышает ожидаемую продолжительность жизни женщин с низким образовательным статусом. Полученные результаты показывают, что в стариих возрастах остается значительная разница в ожидаемой продолжительности жизни в зависимости от образовательного статуса.
\end{abstract}

Ключевые слова: ожидаемая продолжительность жизни, таблицы смертности, модель Кокса, образовательный статус, старение населения, Россия.

В России низкая ожидаемая продолжительность жизни остается одной из актуальных демографических проблем. Существующая демографическая динамика связана с увеличением доли населения в старших возрастных когортах. Важно отметить, что параллельно с этим процессом будет изменяться и образовательный статус пожилого населения, т.к. в старшие возраста будут входить когорты с все более высоким уровнем образования - эта тенденция хорошо видна на данных всероссийских переписей [Население России 2006]. В данной работе мы используем обширные микроданные [РМЭЗ НИУ ВШЭ 2016], чтобы проанализировать различия в модели смертности россиян старше 50 лет в зависимости от уровня образования за последние два десятилетия.

СЕРГЕЙ ГЕОРГИЕВИЧ ШУЛЬГИН (sergey@shulgin.ru), РОССИЙСКАЯ АКАДЕМИЯ НАРОДНОГО ХОЗЯЙСТВА И ГОСУДАРСТВЕННОЙ СЛУЖБЫ ПРИ ПРЕЗИДЕНТЕ РФ, РОССИЯ.

ЮЛИЯ ВИКТОРОВНА ЗИНЬКИНА, МОСКОВСКИЙ ГОСУДАРСТВЕННЫЙ УНИВЕРСИТЕТ ИМ. М.В. ЛОМОНОСОВА, РОССИЙСКАЯ АКАДЕМИЯ НАРОДНОГО ХОЗЯЙСТВА И ГОСУДАРСТВЕННОЙ СЛУЖБЫ ПРИ ПРЕЗИДЕНТЕ РФ, РОССИЯ.

СЕРГЕЙ ЯКОВЛЕВИЧ ЩЕРБОВ, МЕЖДУНАРОДНЫЙ ИНСТИТУТ ПРИКЛАДНОГО СИСТЕМНОГО АНАЛИЗА (IIASA), АвстРия.

ИССЛЕДОВАНИЕ ВЫПОЛНЕНО ПРИ ПОДДЕРЖКЕ РОССИЙСКОГО НАУЧНОГО ФОНДА (ГРАНТ № 17-78-20096).

СТАТЬЯ ПОСТУПИЛА В РЕДАКЦИЮ В ЯНВАРЕ 2018 Г. 
Вопросу влияния различных уровней образования на показатели смертности и ожидаемой продолжительности жизни населения за последние несколько десятилетий было посвящено множество исследований, изучавших в основном развитые страны (например, [Kitagawa, Hauser 1973; Preston, Elo 1995; Mackenbach et al. 1997; Lleras-Muney 2005; Meara, Richards, Cutler 2008]), но также в ряде случаев и развивающиеся государства (например, [Behm 1980; Caldwell 1979; Luo, Zhang, Gu 2015]). В особую группу следует выделить работы, посвященные постсоциалистическим государствам, в особенности России, где динамика смертности и ОПЖ была значительно более неровной, чем в большинстве развитых государств, и отразила в себе масштабные социальные, экономические и политические пертурбации, через который прошла эта группа стран.

Прежде чем перейти к обзору исследований влияния образования на показатели смертности населения России, рассмотрим вкратце общую динамику ОПЖ мужчин и женщин в нашей стране во второй половине XX - начале XXI в. Значительные успехи в повышении ОПЖ были достигнуты в первые десятилетия после окончания Великой Отечественной войны. К 1965 г. ОПЖ мужчин в России составила 64,3 г. (для сравнения во Франции 67,5 г., а в США 66,8 г.), ОПЖ женщин - 73,4 г. (против 74,7 и 73,7 соответственно). Однако начиная со второй половины 1960-х гг. смертность в России прекратила снижаться, а для некоторых групп населения даже стала повышаться. Действительно, потенциал снижения смертности от инфекционных болезней за счет распространения антибиотиков был уже практически исчерпан; дальнейшее ее снижение требовало эффективной борьбы с сердечно-сосудистыми заболеваниями и новообразованиями. Западные страны активно включились в эту борьбу, Россия же заметно отставала [Shkolnikov, Meslé, Vallin, 1996]. По данным Росстата, за период с 1970/71 по 1980/81 гг. ОПЖ мужчин снизилась с 63,21 до 61,53 лет, а ОПЖ женщин - с 73,55 до 73,09 лет [Росстат 2017]. Значительные, но кратковременные улучшения были достигнуты в ходе горбачевской антиалкогольной кампании. Однако они были нивелированы распадом СССР и последовавшим за этим колоссальным скачком смертности; всего за пять лет (с 1990 по 1995 гг.) ОПЖ мужчин обвалилась с 63,73 до 58,12 г., т.е. более чем на 5,5 лет; снижение ОПЖ женщин также было драматическим - с 74,30 до 71,59 г. [Росстат 2017]. Затем в течение 10 лет ОПЖ флуктуировала вокруг этих значений; устойчивый повышательный тренд начал прослеживаться лишь с 2005 г. За последние годы ОПЖ довольно заметно выросла - с 58,92 лет в 2005 г. до 66,5 лет в 2016 г. для мужчин и с 72,47 до 77,06 лет для женщин [Росстат 2017].

Рассмотрим, как динамика ОПЖ преломлялась для групп населения с различными уровнями образования. Исследование Е.М. Андреева и коллег показало, что повышение смертности в 1965-1979 гг. наблюдалось в основном среди работников, занятых физическим трудом, которые в основном имели более низкий уровень образования, чем работники умственного труда [Andreev et al. 2009]. Используя кросс-секционные данные, B.M. Школьников с коллегами проанализировали градиенты смертности в зависимости от продолжительности образования для двух временных точек, привязанных к переписям 1979 и 1989 гг. Их исследование выявило особенно сильное влияние образования на различия в смертности от несчастных случаев и насилия - и у мужчин, и у женщин с начальным или базовым средним образованием смертность от этих причин более чем вдвое превышала 
аналогичные показатели для лиц с более высокими уровнями образования. Еще более заметным был разрыв между людьми с высоким и низким уровнями образования по показателям смертности от причин, связанным с алкоголем [Shkolnikov et al. 1998].

После распада СССР многие постсоциалистические страны Центральной и Восточной Европы пережили период увеличения разрыва между показателями ОПЖ для групп населения с разными уровнями образования (например, [Leinsalu et al. 2008]). Это утверждение подтверждается расчетами В.M. Школьникова и коллег, рассчитавших таблицы смертности для трех образовательных категорий - лиц с высшим образованием, лиц со средним образованием и лиц, имеющих менее чем полное среднее образование. К примеру, в Финляндии и Чехии ОПЖ улучшилась для всех образовательных групп и разрыв между ними увеличился лишь слегка (за счет того, что у высокообразованных слоев ОПЖ росла несколько быстрее, чем у низкообразованных). В Эстонии падение ОПЖ среди лиц с низким и средним уровнем образования в значительной степени компенсировалось ее ростом для групп населения с высоким уровнем образования [Shkolnikov et al. 2006]. В России же наблюдалась значительно более драматическая ситуация: если в 1980 г. ОПЖ мужчин с университетским образованием в возрасте 20 лет превосходила таковую у мужчин, имеющих лишь начальное образование, всего на три года, то к 2001 г. этот разрыв увеличился до 11 лет [Murphy et al. 2006]. Другое исследование, изучавшее различия в ОПЖ в возрасте 21 года между управленцами и специалистами, с одной стороны, и работниками физического труда, с другой, также оценило разрыв между этими категориями примерно в 10 лет [Bessudnov, McKee, Stuckler 2011].

В целом на общероссийском уровне потери низкообразованных слоев населения в ОПЖ были лишь в малой степени компенсированы за счет высокообразованных слоев, что и привело к обвальному падению общей ОПЖ [Shkolnikov et al. 2006]. В особенно уязвимом положении оказались именно мужчины с низким уровнем образования; в возрастной группе 20-39 лет они страдали от чрезвычайно высокой насильственной смертности, а в группе 4059 лет - от очень высокой смертности от сердечно-сосудистых заболеваний [Shkolnikov et al. 1998; Cornia, Paniccia 2000].

Разрыв по показателю ОПЖ между низкообразованными и высокообразованными группами населения достаточно ярко выражен в России и по сей день. В частности, одно из недавних обследований населения показало, что дополнительный год образования примерно на 5\% снижает риск смертности от сердечно-сосудистых заболеваний [Todd, Shkolnikov, Goldman 2016].

\section{ДАННЫЕ И МЕТОДЫ}

Для оценки влияния образовательного статуса на ожидаемую продолжительность жизни мы оцениваем таблицы смертности. Чтобы учесть образовательный статус требуется оценить таблицы смертности для групп населения с различным уровнем образования.

Для решения этой задачи мы используем данные лонгитюдного обследования [РМЭЗ НИУ ВШЭ 2016], которое содержит информацию об уровне образования, годах 
обучения респондента, возрасте, а также позволяет анализировать события, связанные со смертью респондентов.

Для оценки таблиц смертности мы проанализировали 52523 индивида в базе данных РМЭЗ НИУ ВШЭ (волны с 5 по 24-ю), среди которых мы выявили 3276 событий, связанных со смертью индивида, которые удалось связать с индивидуальной анкетой респондента. Мы оценивали таблицы смертности в возрастах от 50 лет с помощью модели смертности Гомперца - Мейкема [Gompertz 1825; Shklovskii 2005]. Среди респондентов РМЭЗ НИУ ВШЭ отобрали 2477 зарегистрированных событий смерти и 13968 дожитий (в возрастах от 50 лет).

Для каждого респондента мы регистрировали дату вхождения, когда информация об этом респонденте в первый раз попала в обследование. Кроме того, для каждого респондента мы зарегистрировали момент его последнего интервью. Событие смерти респондента регистрируется со слов членов его семьи (или домохозяйства). Мы оценивали дату смерти респондента на середине интервала от последнего интервью респондента и первой датой (когда таких несколько) получения информации о смерти респондента от членов его семьи (или домохозяйства).

\section{ПОЛУЧЕННЫЕ ОЦЕНКИ ТАБЛИЦ СМЕРТНОСТИ}

Полученные интервалы дожития и события о смерти индивидов использовались для оценки базового риска. Отдельно для мужчин и женщин мы оценивали модель пропорционального риска [Cox 1972], в которой базовый уровень риска $\lambda_{0}(\mathrm{t})$ описывается функцией Гомперца, а дополнительной объясняющей переменной является возраст. С использованием оценок максимального правдоподобия мы получили оценки параметров $\mathrm{mx}$ и оценили таблицы смертности в возрастах от 50 лет отдельно для мужчин и женщин (таблицы 1 и 2) 1 .

Таблицы смертности оценивались для однолетних возрастных групп, а в таблицах 1 и 2 мы приводим оценки для возрастов от 50 до 110+ лет. Для оцененных таблиц смертности мы привели данные по столбцам: $m_{x}, q_{x}, l_{x}$ и $e_{x}$, где:

$m_{x}-$ возрастной коэффициент смертности;

$q_{x}$ - вероятность смерти на интервале $x, x+1$;

$l_{x}$ - вероятность дожития до возраста $x$ (от 50 лет);

$e_{x}-$ ожидаемая продолжительность жизни (в годах) для данного возраста.

\footnotetext{
${ }^{1}$ Подробные данные таблиц смертности, включая оценки $r_{x}$ из модели Кокса, доступны в формате csv: http://shulgin.ru/files/2018/rlms_lt/lt_rlms_estimates_1994_2014_ssz.csv в формате Excel: http://shulgin.ru/files/2018/rlms_lt//lt_rlms_estimates_1994_2014_ssz.xlsx
} 
Таблица 1. Таблица смертности, оцененная по микроданным РМЭЗ НИУ ВШЭ (волны 5-24), для женщин от 50 лет

\begin{tabular}{c|c|c|c|c}
\hline Возраст & $m_{x}$ & $q_{x}$ & $l_{x}$ & $e_{x}$ \\
\hline 50 & 0,0019 & 0,0019 & 1,0000 & 31,50 \\
55 & 0,0033 & 0,0033 & 0,9882 & 26,84 \\
60 & 0,0060 & 0,0060 & 0,9672 & 22,36 \\
65 & 0,0108 & 0,0107 & 0,9307 & 18,14 \\
70 & 0,0194 & 0,0192 & 0,8684 & 10,77 \\
75 & 0,0349 & 0,0343 & 0,7667 & 7,82 \\
80 & 0,0628 & 0,0609 & 0,6128 & 5,42 \\
85 & 0,1130 & 0,1070 & 0,4095 & 3,58 \\
90 & 0,2033 & 0,1845 & 0,1982 & 2,27 \\
95 & 0,3656 & 0,3091 & 0,0534 & 1,38 \\
100 & 0,6575 & 0,4948 & 0,0049 & 0,82 \\
$110+$ & 1,1825 & 0,7431 & 0,0001 & 0,47 \\
\hline
\end{tabular}

Расчеты авторов на основе данных [РМЭЗ НИУ ВШЭ 2016].

Таблица 2. Таблица смертности, оцененная по микроданным РМЭЗ НИУ ВШЭ (волны 5-24), для мужчин от 50 лет

\begin{tabular}{c|c|c|c|c}
\hline Возраст & $m_{x}$ & $q_{x}$ & $l_{x}$ & $e_{x}$ \\
\hline 50 & 0,0118 & 0,0118 & 1,0000 & 21,17 \\
55 & 0,0180 & 0,0179 & 0,9319 & 17,53 \\
60 & 0,0274 & 0,0271 & 0,8371 & 11,28 \\
65 & 0,0418 & 0,0409 & 0,7108 & 8,74 \\
70 & 0,0637 & 0,0617 & 0,5540 & 6,62 \\
75 & 0,0970 & 0,0925 & 0,3791 & 4,89 \\
80 & 0,1477 & 0,1376 & 0,2125 & 3,53 \\
85 & 0,2250 & 0,2023 & 0,0879 & 2,50 \\
90 & 0,3427 & 0,2926 & 0,0228 & 1,74 \\
105 & 0,5220 & 0,4140 & 0,0029 & 1,19 \\
105 & 0,7952 & 0,5690 & 0,0001 & 0,81 \\
$110+$ & 1,2112 & 0,7544 & 0,0000 & 0,54 \\
\hline
\end{tabular}

Для оценки качества полученных результатов мы провели оценку доверительных интервалов распределения полученных оценок. Оценка параметров модели пропорциональных рисков Кокса выполняется с использованием функции нелинейной оптимизации optim из программного пакета R [R Core Team, 2017] и применения алгоритма Нельдера-Мида [Nelder, Mead, 1965] для нахождения оптимума. В оптимуме мы также получаем численные оценки дифференцированной матрицы Гесса 2 . Обратная матрица Гесса является оценкой вариационно-ковариационной матрицы оценок.

Чтобы перевести неопределенность в оцененных параметрах в неопределенность полученных оценок ожидаемой продолжительности жизни мы используем метод моделирования Монте-Карло для генерации множественных коррелированных переменных. Применяя разложение Холецкого к полученной вариационноковариационной матрице оценок, мы можем получить нижнюю треугольную матрицу V. Создавая некоррелированные нормально распределенные выборки х и применяя V к x, мы

\footnotetext{
${ }^{2}$ Подробнее см. описание работы R функции орtim https://stat.ethz.ch/R-manual/R-devel/library/stats/html/optim.html 
создаем выборку вектора $\mathrm{V}_{\mathrm{x}}$, который обладает ковариационными свойствами оцененных параметров. Используя полученные $\mathrm{V}_{\mathrm{x}}$ в качестве $\mathrm{m}_{\mathrm{x}}$, мы оцениваем значения ожидаемой продолжительности жизни. Полученные таким образом различные значения ожидаемой продолжительности жизни мы используем для оценки доверительных интервалов интересующих нас параметров. В данной работе мы приводим оценки 95\% доверительных интервалов.

Полученные в таблицах 1 и 2 оценки таблиц смертности характеризуют выборку лонгитюдного обследования РМЭЗ НИУ ВШЭ в старших возрастах на длительном интервале времени - с 1994 по 2015 г. За это время Россия переживала как периоды сокращения ОПЖ, так и периоды быстрого роста. Для сопоставления сравним полученные оценки с данными Human Mortality Database [HMD 2016] (таблица 3).

Таблица 3. Оценки ОПЖ в возрастах от 50 до 75 лет по данными НМD и по оценкам на основе выборки РМЭЗ НИУ ВШЭ (лет)

\begin{tabular}{|c|c|c|c|c|c|c|c|c|}
\hline \multirow[b]{2}{*}{$\begin{array}{l}\tilde{0} \\
\tilde{\tilde{O}} \\
\tilde{0} \\
\stackrel{0}{0}\end{array}$} & \multicolumn{6}{|c|}{ ОПЖ по 1х10-летним таблицам смертности HMD } & \multicolumn{2}{|c|}{$\begin{array}{c}\text { Оценки ОПЖ по РМЭЗ НИУ ВШЭ } \\
(1994-2015 \text { гг.) * }\end{array}$} \\
\hline & 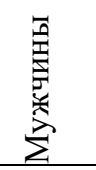 & $\begin{array}{l}\text { 自 } \\
\text { 罵 } \\
\text { 突 }\end{array}$ & 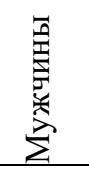 & 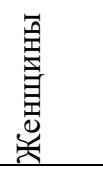 & 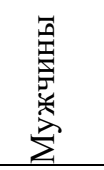 & 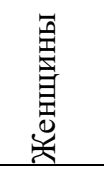 & Мужчины & Женщины \\
\hline 50 & 19,50 & 26,99 & 19,00 & 27,07 & 21,66 & 29,26 & $21,2(20,6-21,7)$ & $31,5(31-32)$ \\
\hline 55 & 16,41 & 22,84 & 16,13 & 23,00 & 18,30 & 24,99 & $17,5(17,1-18)$ & $26,8(26,4-27,3)$ \\
\hline 60 & 13,64 & 18,90 & 13 & 19 , & 15 & 20,92 & $14,2(1$ & $22,4(22$ \\
\hline 65 & 11,15 & 15,21 & 11,15 & 15, & 12,66 & 17,06 & $11,3(10$ & $18,1(17,8$ \\
\hline 70 & 8,99 & 11,86 & 9,01 & 12,08 & 10,18 & 13,42 & $8,7(8,4-9,1)$ & $14,2(13,9-14,6)$ \\
\hline 75 & 7,03 & 8,95 & 7,14 & 9,09 & 8,07 & 10,14 & $6,6(6,3-6,9)$ & $10,8(10,4-11,1)$ \\
\hline 80 & 5,28 & 6,49 & 5,53 & 6,64 & 6,27 & 7,38 & $4,9(4,6-5,2)$ & $7,8(7,5-8,1)$ \\
\hline
\end{tabular}

Примечания: * в скобках приведены 95\% доверительные интерваль полученных оценок.

Из таблицы 3 видно, что в целом оценки ОПЖ для мужчин, полученные по выборке РМЭЗ НИУ ВШЭ, оказываются сопоставимыми (с учетом динамики оценок ОПЖ с 1994 по 2015 гг.) с оценками, полученными на общестрановой статистике. Для женщин оценки ОПЖ по выборке РМЭЗ НИУ ВШЭ оказываются несколько выше, чем оценки по общестрановой статистике, представленные в базе данных Human Mortality Database.

Для анализа влияния уровня образования на модель смертности мы оценили таблицы смертности для лиц с различной продолжительностью обучения. В качестве порогового значения была выбрана продолжительность обучения в 10 лет. К группе лиц с высоким образовательным статусом мы отнесли тех, у кого длительность обучения превышает 10 $л^{3}{ }^{3}$. Как правило, представители этой группы имеют законченное высшее или среднее специальное образование. К группе с низким образовательным статусом мы отнесли лиц с продолжительностью обучения 10 и менее лет.

3 Для классификации мы использовали следующие категории: «есть диплом о высшем образовании», «аспирантура и т.п. с дипломом», «аспирантура и т.п. без диплома», «10 и более классов школы и техникум без диплома», «техникум с дипломом». 
Для оценки влияния образовательного статуса мы оценивали модель пропорционального риска [Сох 1972], в которой базовый уровень риска $\lambda o(t)$ описывается функцией Гомперца, а дополнительными объясняющими переменными являются возраст и образовательный статус, которые и определяли пропорциональный (относительно базового уровня) риск.

С использованием оценок максимального правдоподобия (для мужчин и женщин оценивались свои модели) мы получили оценки параметров $m_{x}$ и для каждого образовательного статуса оценили таблицы смертности в возрастах от 50 лет отдельно для мужчин и женщин (таблицы 4-7).

Таблица 4. Таблица смертности, оцененная по микроданным РМЭЗ НИУ ВШЭ (волны 5-24), для мужчин от 50 лет с высоким образовательным статусом

\begin{tabular}{c|c|c|cc}
\hline Возраст & $m_{x}$ & $q_{x}$ & $l_{x}$ & $e_{x}$ \\
\hline 50 & 0,0102 & 0,0102 & 1,0000 & 22,80 \\
55 & 0,0154 & 0,0153 & 0,9412 & 19,06 \\
60 & 0,0233 & 0,0230 & 0,8589 & 12,64 \\
65 & 0,0351 & 0,0345 & 0,7481 & 9,88 \\
70 & 0,0530 & 0,0516 & 0,6074 & 7,59 \\
75 & 0,0799 & 0,0769 & 0,4435 & 5,71 \\
80 & 0,1206 & 0,1138 & 0,2759 & 4,19 \\
85 & 0,1820 & 0,1669 & 0,1347 & 3,02 \\
90 & 0,2747 & 0,2415 & 0,0455 & 2,13 \\
95 & 0,4146 & 0,3434 & 0,0088 & 1,48 \\
100 & 0,6256 & 0,4765 & 0,0007 & 1,02 \\
105 & 0,9440 & 0,6413 & 0,0000 & 0,70 \\
\hline
\end{tabular}

Источник: расчеты авторов на данных [РМЭЗ НИУ ВШЭ 2016].

Таблица 5. Таблица смертности, оцененная по микроданным РМЭЗ НИУ ВШЭ (волны 5-24), для мужчин от 50 лет с низким образовательным статусом

\begin{tabular}{c|c|c|c|c}
\hline Возраст & $m_{x}$ & $q_{x}$ & $l_{x}$ & $e_{x}$ \\
\hline 50 & 0,0141 & 0,0140 & 1,0000 & 16,85 \\
55 & 0,0213 & 0,0210 & 0,9197 & 13,20 \\
60 & 0,0321 & 0,0316 & 0,8107 & 10,43 \\
65 & 0,0484 & 0,0473 & 0,6700 & 8,06 \\
70 & 0,0731 & 0,0705 & 0,5026 & 6,08 \\
75 & 0,1103 & 0,1045 & 0,3256 & 3,49 \\
80 & 0,1665 & 0,1537 & 0,1690 & 2,30 \\
85 & 0,2512 & 0,2232 & 0,0627 & 1,61 \\
90 & 0,3791 & 0,3187 & 0,0140 & 1,11 \\
100 & 0,5720 & 0,4448 & 0,0014 & 0,75 \\
105 & 0,8632 & 0,6029 & 0,0000 & 0,51 \\
$110+$ & 1,3026 & 0,7888 & 0,0000 & 0,0000 \\
\hline
\end{tabular}

Источник: расчеты авторов на данных [РМЭЗ НИУ ВШЭ 2016]. 
Таблица 6. Таблица смертности, оцененная по микроданным РМЭЗ НИУ ВШЭ (волны 5-24), для женщин от 50 лет с высоким образовательным статусом

\begin{tabular}{ccc|c|c}
\hline Возраст & $m_{x}$ & $q_{x}$ & $l_{x}$ & $e_{x}$ \\
\hline 50 & 0,0015 & 0,0015 & 1,0000 & 29,45 \\
55 & 0,0027 & 0,0027 & 0,9904 & 25,22 \\
60 & 0,0046 & 0,0046 & 0,9739 & 20,89 \\
65 & 0,0081 & 0,0080 & 0,9458 & 16,84 \\
70 & 0,0141 & 0,0140 & 0,8988 & 13,16 \\
75 & 0,0245 & 0,0242 & 0,8222 & 9,92 \\
80 & 0,0428 & 0,0419 & 0,7040 & 7,19 \\
85 & 0,0746 & 0,0719 & 0,5370 & 5,00 \\
90 & 0,1301 & 0,1222 & 0,3349 & 3,34 \\
95 & 0,2269 & 0,2038 & 0,1468 & 2,14 \\
100 & 0,3957 & 0,3303 & 0,0346 & 1,33 \\
$110+$ & 0,6900 & 0,5130 & 0,0027 & 0,83 \\
\hline
\end{tabular}

Источник: расчеты авторов на данных [РМЭЗ НИУ ВШЭ 2016].

Таблица 7. Таблица смертности, оцененная по микроданным РМЭЗ НИУ ВШЭ (волны 5-24), для женщин от 50 лет с низким образовательным статусом

\begin{tabular}{c|c|c|c|c}
\hline Возраст & $\mathrm{mx}$ & $\mathrm{qx}$ & $\mathrm{lx}$ & $\mathrm{ex}$ \\
\hline 50 & 0,0026 & 0,0026 & 1,0000 & 30,06 \\
55 & 0,0045 & 0,0045 & 0,9839 & 25,50 \\
60 & 0,0078 & 0,0078 & 0,9565 & 17,16 \\
65 & 0,0136 & 0,0135 & 0,9105 & 13,38 \\
70 & 0,0237 & 0,0234 & 0,8356 & 10,11 \\
75 & 0,0413 & 0,0405 & 0,7194 & 7,35 \\
80 & 0,0720 & 0,0695 & 0,5540 & 5,12 \\
85 & 0,1256 & 0,1182 & 0,3512 & 3,43 \\
90 & 0,2190 & 0,1974 & 0,1585 & 2,21 \\
15 & 0,3819 & 0,3206 & 0,0393 & 1,37 \\
105 & 0,6658 & 0,4995 & 0,0033 & 0,83 \\
$110+$ & 1,1610 & 0,7346 & 0,0000 & 0,49 \\
\hline
\end{tabular}

Источник: расчеты авторов на данных [РМЭЗ НИУ ВШЭ 2016].

\section{ЗАКЛЮЧЕНИЕ}

Оценки таблиц смертности, проведенные по выборке РМЭЗ НИУ ВШЭ в интервале с 1994 по 2015 г., подтверждают существующие данные о том, что образовательный статус влияет на модель смертности и ожидаемую продолжительность жизни. Предыдущие исследования показывают, что значительные различия в ОПЖ в зависимости от образовательного статуса возникают в трудоспособных возрастах и во многом связаны с внешними причинами смерти (насильственными смертями, потреблением алкоголя и т.п.). Как видно из таблиц 8 и 9, в старших возрастах остается значительная разница в ОПЖ в зависимости от образовательного статуса. 
Таблица 8. Различия в ОПЖ мужчин с высоким и низким образовательным статусом (лет)

\begin{tabular}{c|c|c|c|c}
\hline Возраст & ОПЖ в целом & $\begin{array}{c}\text { ОПЖ с высоким } \\
\text { образовательным } \\
\text { статусом }\end{array}$ & $\begin{array}{c}\text { ОПЖ с низким } \\
\text { образовательным } \\
\text { статусом }\end{array}$ & $\begin{array}{c}\text { Разница в ОПЖ } \\
\text { между высоким и } \\
\text { низким }\end{array}$ \\
\hline 50 & $21,2(20,6-21,7)$ & $22,8(22-23,6)$ & $19,9(19,2-20,5)$ & $3,0(1,9-4,1)$ \\
55 & $17,5(17,1-18)$ & $19,1(18,3-19,8)$ & $16,3(15,8-16,9)$ & $2,7(1,7-3,7)$ \\
60 & $14,2(13,8-14,6)$ & $15,6(15-16,4)$ & $13,2(12,7-13,7)$ & $2,4(1,6-3,3)$ \\
65 & $11,3(10,9-11,6)$ & $12,6(12-13,2)$ & $10,4(10-10,8)$ & $2,1(1,4-2,9)$ \\
70 & $8,7(8,4-9,1)$ & $9,9(9,3-10,5)$ & $8,1(7,6-8,4)$ & $1,8(1,2-2,5)$ \\
85 & $6,6(6,3-6,9)$ & $7,6(7,1-8,2)$ & $6,1(5,7-6,4)$ & $1,5(1-2,1)$ \\
80 & $4,9(4,6-5,2)$ & $5,7(5,2-6,2)$ & $4,5(4,2-4,8)$ & $1,2(0,8-1,7)$ \\
\hline
\end{tabular}

Таблица 9. Различия в ОПЖ женщин с высоким и низким образовательным статусом (лет)

\begin{tabular}{c|c|c|c|c}
\hline Возраст & ОПЖ целом & $\begin{array}{c}\text { ОПЖ с высоким } \\
\text { образовательным } \\
\text { статусом }\end{array}$ & $\begin{array}{c}\text { ОПЖ с низким } \\
\text { образовательным } \\
\text { статусом }\end{array}$ & $\begin{array}{c}\text { Разница в ОПЖ } \\
\text { между высоким и } \\
\text { низким }\end{array}$ \\
\hline 50 & $31,5(31-32)$ & $34,5(33,4-35,6)$ & $30,1(29,4-30,7)$ & $4,4(3,1-5,8)$ \\
55 & $26,8(26,4-27,3)$ & $29,8(28,7-30,9)$ & $25,5(24,9-26,1)$ & $4,3(3-5,6)$ \\
60 & $22,4(22-22,8)$ & $25,2(24,2-26,3)$ & $21,2(20,6-21,7)$ & $4,1(2,9-5,3)$ \\
65 & $18,1(17,8-18,5)$ & $20,9(19,9-21,9)$ & $17,1(16,6-17,6)$ & $3,8(2,7-5)$ \\
70 & $14,2(13,9-14,6)$ & $16,8(15,9-17,8)$ & $13,4(13-13,8)$ & $3,5(2,4-4,5)$ \\
75 & $10,8(10,4-11,1)$ & $13,2(12,3-14,1)$ & $10,1(9,7-10,5)$ & $3,1(2,1-4)$ \\
80 & $7,8(7,5-8,1)$ & $9,9(9,1-10,8)$ & $7,4(7-7,7)$ & $2,6(1,8-3,4)$ \\
\hline
\end{tabular}

В возрасте 50 лет ожидаемая продолжительность жизни мужчин с высоким образовательным статусом оказывается на три года выше, чем для мужчин с низким образовательным статусом. Для женщин эти различия ещё более значимы: в возрасте 50 лет ОПЖ для женщин с высоким образовательным статусом оказывается выше на 4,4 года, чем для женщин с низким образовательным статусом.

\section{ЛИТЕРАТУРА}

Население России 2003-2004. Одиннадцатый-двенадцатый ежегодный демографический доклад / Под ред. Вишневского А.Г. М.: "Наука", 2006.

РМЭЗ НИУ ВШЭ (2016). Российский мониторинг экономического положения и здоровья населения НИУ-ВШЭ (RLMS-HSE), проводимый Национальным исследовательским университетом "Высшая школа экономики" и ООО «Демоскоп» при участии Центра народонаселения Университета Северной Каролины в Чапел Хилле и Института социологии PAH. URL: http://www.cpc.unc.edu/projects/rlms/; http://www.hse.ru/rlms (данные загружены 05.09.2016).

Росстат (2017). Ожидаемая продолжительность жизни при рождении. М.: Федеральная служба государственной статистики. URL:

http://www.gks.ru/free_doc/new_site/population/demo/demo26.xlsx (данные загружены 25.09.2017).

Andreev E., R. Hoffmann, E. Carlson, V. Shkolnikov, T. L. Kharkova (2009). Concentration of working-age male mortality among manual workers in urban Latvia and Russia, 1970-1989 // European societies. 11(1): 161-185. 
Behm H. (1980). Socio-economic determinants of mortality in Latin America // Population bulletin. 13: 1-15.

Bessudnov A., M. McKee, D. Stuckler (2011). Inequalities in male mortality by occupational class, perceived status and education in Russia, 1994-2006 // The European journal of public health. 22(3): 332-337.

Caldwell J. C. (1979). Education as a factor in mortality decline an examination of Nigerian data // Population studies. 33(3): 395-413.

Cornia G. A., R. Paniccià (2000). The Mortality Crisis in Transitional Economies. Oxford: Oxford University Press. 480 p.

Cox D. (1972). Regression Models and Life-Tables // Journal of the Royal Statistical Society, Series B. 34 (2): 187-220.

Gompertz B. (1825). On the nature of the function expressive of the law of human mortality, and on a new mode of determining the value of life contingencies // Philosophical Transactions of the Royal Society of London. 115: 513-583.

HMD (2017). The Human Mortality Database. University of California, Berkeley (USA), and Max Planck Institute for Demographic Research (Germany). URL: http://www.mortality.org/; http://www.humanmortality.de/ (downloaded 20.09.2017).

Kitagawa E. M., P. M. Hauser (1973). Differential mortality in the United States: a study in socioeconomic epidemiology. Cambridge, MA: Harvard University Press. 255 p.

Leinsalu M., I. Stirbu, D. Vågerö, R. Kalėdienė, K. Kovács, B. Wojtyniak, W. Wroblewska, J.P. Mackenbach, A.E. Kunst (2008). Educational inequalities in mortality in four Eastern European countries: divergence in trends during the post-communist transition from 1990 to 2000 // International Journal of Epidemiology. 38(2): 512-525.

Lleras-Muney A. (2005). The relationship between education and adult mortality in the United States // The Review of Economic Studies. 72(1): 189-221.

Luo Y., Z. Zhang, D. Gu (2015). Education and mortality among older adults in China // Social Science \& Medicine. 127: 134-142.

Mackenbach J. P., A. E. Kunst, A. E. Cavelaars, F. Groenhof, J. J. Geurts, EU Working Group on Socioeconomic Inequalities in Health (1997). Socioeconomic inequalities in morbidity and mortality in Western Europe // The Lancet. 349 (9066): 1655-1659.

Meara E. R., S. Richards, D. M. Cutler (2008). The gap gets bigger: changes in mortality and life expectancy, by education, 1981-2000 // Health Affairs. 27(2): 350-360.

Murphy M., M. Bobak, A. Nicholson, R. Rose, M. Marmot (2006). The widening gap in mortality by educational level in the Russian Federation, 1980-2001 // American Journal of Public Health. 96(7): 1293-1299.

Nelder, J. A. and Mead, R. (1965) A simplex algorithm for function minimization // Computer Journal 7, 308-313.

Preston S.H., I.T. Elo (1995). Are educational differentials in adult mortality increasing in the United States? // Journal of Aging and Health. 7(4): 476-496.

R Core Team (2017). R: A language and environment for statistical computing. R Foundation for Statistical Computing, Vienna, Austria. URL https://www.R-project.org/. 
RLMS-HSE (2016). Russian Longitudinal Monitoring Survey - Higher School of Economics. URL: http://www.cpc.unc.edu/projects/rlms/; http://www.hse.ru/rlms (data downloaded on 05.09.2016).

Rosstat (2017). Life expectancy at birth. Moscow: Russian Federation Federal State Statistics Service. URL: http://www.gks.ru/free_doc/new_site/population/demo/demo26.xlsx (data downloaded on 25.09.2017).

Shklovskii B. I. (2005). A simple derivation of the Gompertz law for human mortality // Theory in Biosciences. 123(4): 431-433.

Shkolnikov V. M., D. A. Leon, S. Adamets, E. Andreev, A. Deev (1998). Educational level and adult mortality in Russia: an analysis of routine data 1979 to 1994 // Social Science \& Medicine. 47(3): 357-369.

Shkolnikov V. M., E. M. Andreev, D. Jasilionis, M. Leinsalu, O.I. Antonova, M. McKee (2006). The changing relation between education and life expectancy in central and eastern Europe in the 1990s // Journal of Epidemiology \& Community Health. 60(10): 875-881.

Shkolnikov V., F. Meslé, J. Vallin (1996). Health crisis in Russia // Population: an English selection. 8: 123-190.

Todd M. A., V. M. Shkolnikov, N. Goldman (2016). Why are well-educated Muscovites more likely to survive? Understanding the biological pathways // Social Science \& Medicine. 157: 138-147. 


\title{
LIFE EXPECTANCY OF ELDERLY IN RUSSIA DEPENDING ON EDUCATIONAL STATUS
}

\author{
SERGEY SHULGIN, JULIA ZINKINA, SERGEI SCHERBOV
}

\begin{abstract}
Russia is currently experiencing an increase in the proportion of the population belonging to senior age cohorts. This process is paralleled by changes in the educational structure, as comparatively more educated groups enter senior ages. In this paper we analyze the differences in mortality and life expectancy depending on the level of education for Russians aged 50 and older. We estimate life tables for people at these ages. Our estimates are based on the RLMS-HSE longitudinal survey for the period from 1994 to 2015. We take into account all the waves of the survey from the 5th to the 24th. Life tables are estimated separately for men and women. Apart from general life tables for each sex, we also estimate life tables for people with low educational status and high educational status (also segregated by sex). People with 10 years or less of education are considered to have low educational status, while those with more than 10 years of education are regarded as having high educational status. Our calculations reveal that at the age of 50, men with high educational status have life expectancy values 3 years higher than their counterparts with low educational status. As for women, at the same age of 50, those with high educational status have life expectancy values 4.4 years higher than their counterparts with low educational status. Our results reveal that a remarkable difference still persists in life expectancy values depending on the highest level of education achieved.
\end{abstract}

Key words: life expectancy, life tables, Cox proportional hazard model, educational status, population aging, Russia.

\begin{abstract}
Sergey Shulgin (sergey@shulgin.ru), Russian Presidential Academy of National Economy and Public ADMINISTRATION (RANEPA), RUSSIA.

Julia Zinkina, Lomonosov Moscow State University, Russian Presidential ACademy of National Economy AND PUBLIC ADMINISTRATION (RANEPA), RUSSIA.
\end{abstract}

SERgei Scherbov, InTERnational Institute For ApPlied Systems ANALYSis (IIASA), Austria.

THIS RESEARCH HAS BEEN SUPPORTED BY THE RUSSIAN SCIENCE FOUNDATION, PROJECT No 17-78-20096.

DATE RECEIVED: JANUARY 2018.

\section{REFERENCES}

Andreev E., R. Hoffmann, E. Carlson, V. Shkolnikov, T. L. Kharkova (2009). Concentration of working-age male mortality among manual workers in urban Latvia and Russia, 1970-1989 // European societies. 11(1): 161-185.

Behm H. (1980). Socio-economic determinants of mortality in Latin America // Population bulletin. 13: 1-15.

Bessudnov A., M. McKee, D. Stuckler (2011). Inequalities in male mortality by occupational class, perceived status and education in Russia, 1994-2006 // The European journal of public health. 22(3): 332-337.

Caldwell J. C. (1979). Education as a factor in mortality decline an examination of Nigerian data // Population studies. 33(3): 395-413.

Cornia G. A., R. Paniccià (2000). The Mortality Crisis in Transitional Economies. Oxford: Oxford University Press. 480 p. 
Cox D. (1972). Regression models and life-tables // Journal of the Royal Statistical Society, Series B. 34 (2): 187-220.

Gompertz B. (1825). On the nature of the function expressive of the law of human mortality, and on a new mode of determining the value of life contingencies // Philosophical Transactions of the Royal Society of London. 115: 513-583.

HMD (2017). The Human Mortality Database. University of California, Berkeley (USA), and Max Planck Institute for Demographic Research (Germany). URL: http://www.mortality.org/; http://www.humanmortality.de/ (downloaded: 20.09.2017).

Kitagawa E. M., P. M. Hauser (1973). Differential mortality in the United States: a study in socioeconomic epidemiology. Cambridge, MA: Harvard University Press. 255 p.

Leinsalu M., I. Stirbu, D. Vågerö, R. Kalėdienė, K. Kovács, B. Wojtyniak, W. Wroblewska, J.P. Mackenbach, A.E. Kunst (2008). Educational inequalities in mortality in four Eastern European countries: divergence in trends during the post-communist transition from 1990 to 2000 // International Journal of Epidemiology. 38(2): 512-525.

Lleras-Muney A. (2005). The relationship between education and adult mortality in the United States // The Review of Economic Studies. 72(1): 189-221.

Luo Y., Z. Zhang, D. Gu (2015). Education and mortality among older adults in China // Social Science \& Medicine. 127: 134-142.

Mackenbach J. P., A. E. Kunst, A. E. Cavelaars, F. Groenhof, J. J. Geurts, EU Working Group on Socioeconomic Inequalities in Health (1997). Socioeconomic inequalities in morbidity and mortality in Western Europe // The Lancet. 349 (9066): 1655-1659.

Meara E. R., S. Richards, D. M. Cutler (2008). The gap gets bigger: changes in mortality and life expectancy, by education, 1981-2000 // Health Affairs. 27(2): 350-360.

Murphy M., M. Bobak, A. Nicholson, R. Rose, M. Marmot (2006). The widening gap in mortality by educational level in the Russian Federation, 1980-2001 // American Journal of Public Health. 96(7): 1293-1299.

Nelder, J. A. and Mead, R. (1965) A simplex algorithm for function minimization // Computer Journal 7, 308-313.

R Core Team (2017). R: A language and environment for statistical computing. R Foundation for Statistical Computing, Vienna, Austria. URL https://www.R-project.org/.

RLMS-HSE (2016). Russia Longitudinal Monitoring survey, conducted by National Research University Higher School of Economics and OOO "Demoscope" together with Carolina Population Center, University of North Carolina at Chapel Hill and the Institute of Sociology of the Federal Center of Theoretical and Applied Sociology of the Russian Academy of Sciences. URL: http://www.cpc.unc.edu/projects/rlms-hse, http://www.hse.ru/org/hse/rlms (accessed 05.09.2016).

Population of Russia [Naseleniye Rossii] 2003-2004 (2006). Eleventh to twelfth annual demographic report [Odinnadtsatyy-dvenadtsatyy yezhegodnyy demograficheskiy doklad] / A.G. Vishnevsky, ed. Moscow: Nauka.

Rosstat (2017). Life expectancy at birth [Ozhidayemaya prodolzhitel'nost' zhizni pri rozhdenii]. Moscow: Federal State Statistics Service [Federal'naya sluzhba gosudarstvennoy statistiki]. URL: http://www.gks.ru/free_doc/new_site/population/demo/demo26.xlsx (accessed 25.09.2017). 
Preston S.H., I.T. Elo (1995). Are educational differentials in adult mortality increasing in the United States? // Journal of Aging and Health. 7(4): 476-496.

Shklovskii B. I. (2005). A simple derivation of the Gompertz law for human mortality // Theory in Biosciences. 123(4): 431-433.

Shkolnikov V. M., D. A. Leon, S. Adamets, E. Andreev, A. Deev (1998). Educational level and adult mortality in Russia: an analysis of routine data 1979 to 1994 // Social Science \& Medicine. 47(3): 357-369.

Shkolnikov V. M., E. M. Andreev, D. Jasilionis, M. Leinsalu, O.I. Antonova, M. McKee (2006). The changing relation between education and life expectancy in central and eastern Europe in the 1990s // Journal of Epidemiology \& Community Health. 60 (10): 875-881.

Shkolnikov V., F. Meslé, J. Vallin (1996). Health crisis in Russia // Population: an English selection. 8: 123-190.

Todd M. A., V. M. Shkolnikov, N. Goldman (2016). Why are well-educated Muscovites more likely to survive? Understanding the biological pathways // Social Science \& Medicine. 157: 138-147. 